\title{
Mesoscale Folding, Instability, and Disruption of Laminar Flow in Metal Surfaces
}

\author{
Narayan K. Sundaram, ${ }^{*}$ Yang Guo, ${ }^{\dagger}$ and Srinivasan Chandrasekar \\ Center for Materials Processing and Tribology, Purdue University, West Lafayette, Indiana 47907-2023, USA
}

(Received 18 June 2012; revised manuscript received 19 July 2012; published 4 September 2012)

\begin{abstract}
Using in situ imaging, we report surface fold formation and fluidlike flow instabilities in sliding of annealed copper. We demonstrate using simulations that folding is principally driven by grain-induced plastic instability. The phenomenon shows remarkable similarities with Kelvin-Helmholtz-type flow instabilities in fluids. While such instabilities have been conjectured to exist in sliding interfaces at the nanoscale, we find vortices and folding in metals at the mesoscale. The occurrence of folds impacts many applications, including surface generation processes and tribology.
\end{abstract}

Sliding metal interfaces are important for the physics of wear [1], friction [2], machining [3], and generation of graded and nanograined structures [4,5]. Using high resolution in situ imaging and direct flow measurement in a relatively unstudied regime $(\approx 100 \mu \mathrm{m}-1 \mathrm{~mm})$ in prior sliding studies [1-6], we report the formation of mesoscale folds and primitive vortical structures at a free surface in sliding of annealed oxygen-free high conductivity copper. We deduce a mechanism for the formation of such folds using simulations. The phenomenon shows remarkable similarities with Kelvin-Helmholtz-type flow instabilities in fluids [7]. Importantly, while such instabilities have been conjectured to exist in sliding interfaces at the nanoscale [8], our experiments show folding in metals at the mesoscale and away from the interface itself. The occurrence of folds impacts many applications, including surface generation processes and tribology. In particular, it may limit the quality of metal surfaces produced by repeated sliding treatments suggested in the past [5].

Our mesoscale sliding system (Fig. S1) consists of an annealed oxygen-free high conductivity copper workpiece (grain size $118 \mu \mathrm{m}$ ) sliding against a hard steel wedge indenter at a (low) velocity of $1 \mathrm{~mm} / \mathrm{sec}$ [9]. Two values of the indenter angle $\alpha, \alpha=-70^{\circ}$ and $\alpha=-60^{\circ}$, are chosen to demonstrate the phenomenon of interest. Particle image velocimetry [10] of the raw image sequence and additional postprocessing [11] provides a comprehensive record of the velocity and strain field histories.

Remarkably, when $\alpha=-60^{\circ}$, the initial polished surface of the workpiece ahead of the wedge face develops a series of protuberances or bumps, which grow in amplitude, interact with one another, and develop into folds, as shown in Fig. 1. The folds and the sinuous nature of the superimposed, near-surface flow or streak lines, obtained from the particle image velocimetry velocity field, indicate breakdown of laminar flow and a drastically different flow pattern than assumed in triboplasticity $[12,13]$. The bumps have an amplitude of 100-200 $\mu \mathrm{m}$ when fully developed. The formation of bumps and folds precedes contact with the wedge face spatially and temporally. A fold does not change much as it traverses the wedge face until it passes the tip. At this stage, it undergoes stretching and rotation into a shallow, long, cracklike feature that is inclined at a very acute angle to the surface of the workpiece. This feature can be inferred from the inflection in the green flowline nearest to the surface, and just past the wedge tip, in frame 3. A video of folding is shown in Movie S1.

Folds can be complicated; a pair of simple folds occasionally comes in contact to produce a complex or multiple fold or primitive vortex structure, as shown in frame 4 of Fig. 1 (also see Fig. S2). The free surface of the workpiece itself [uppermost, thick (white) line in Fig. 1] is demarcated by manually identifying the edge and tracking surface features as they evolve. The presence and location of a simple fold may be determined by tracking minima in the streak line closest to the surface.

In contrast, at $\alpha=-70^{\circ}$ flow is essentially laminar, as indicated by the surface and by a lack of meander in the streak lines in Fig. 2. Minor protuberances that develop ahead of the wedge face do not grow in amplitude or generate significant folds. The angle $\alpha$ is thus a critical parameter in determining the laminarity of plastic flow. The plastic flow is even less sinuous at $\alpha=-80^{\circ}$.

Scanning electron micrographs of the contact region with $\alpha=-60^{\circ}$ are shown in Fig. 3. A low magnification overall view is shown in 3(a). Four distinct types of surface deformation features can be identified at different locations, as shown in high magnification subfigures 3(b)-3(e). The initial smooth surface develops small surface protuberances far away from the wedge face in 3(b). Traveling toward the wedge face, the protuberances are considerably more developed (location c). The extent of the features in 3 (c) is approximately $100 \mu \mathrm{m}$ and resembles grains, suggesting that individual grains undergo differential deformation to produce bumps. Slip bands are seen within grains on the free surface in 3(c). The material in the wake of the wedge appears smooth in 3(a), but, at higher magnification, two distinct types of cracklike features are seen in 3(d) and 3(e). Figure 3(e) shows a fingered crack or fold feature on the specimen surface after material has 

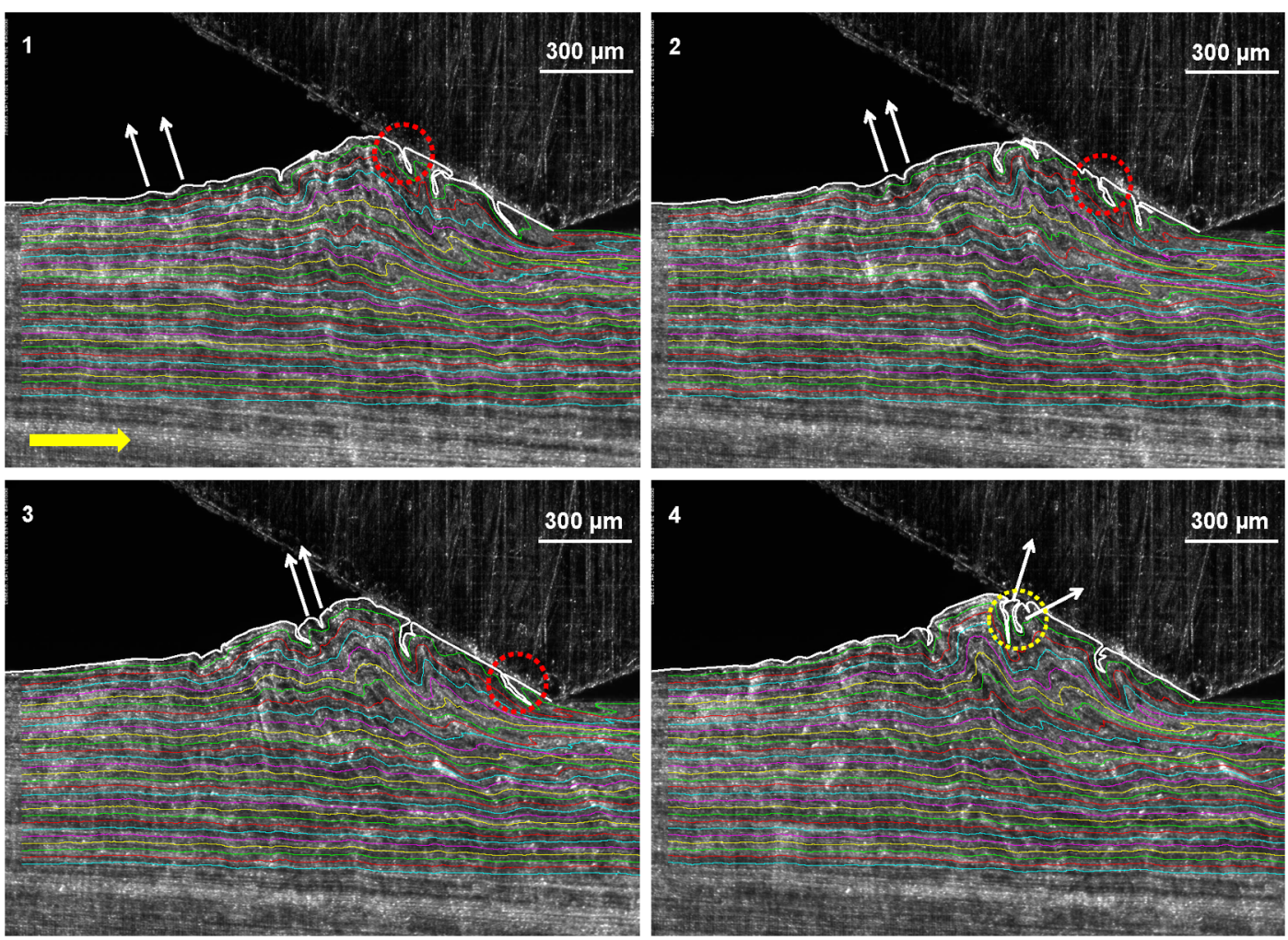

FIG. 1 (color online). Complex, nonlaminar flow and fold formation for $\alpha=-60^{\circ}$. These four frames were selected from an in situ high-speed image sequence to show fold development. Thin white arrows track the evolution of a pair of neighboring bumps generated ahead of the interface as they interact to form a surface fold. The dotted red circles in frames 1-3 track time evolution of an existing fold. The dotted yellow circle in frame 4 highlights a complex fold, one of which was formed by the bumps in frame 1 . The uppermost, thick white line is the manually identified surface. Superimposed, subsurface, colored lines are streak lines produced from velocity measurements. The sliding direction is indicated by the thick yellow arrow. The snapshot times are $t_{1}=7.5 \mathrm{~s}, t_{2}=8.0 \mathrm{~s}, t_{3}=8.5 \mathrm{~s}$, and $t_{4}=9.0 \mathrm{~s}$, covering $1.5 \mathrm{~s}$ of real time.

passed the wedge tip; imaged side-on, this feature appears as a faint, long, shallow feature inclined at an acute angle of 5-10 degrees to the workpiece surface. Figure 3(d) shows a surface tear, also observed in the wake of the wedge. Such tears occur at isolated locations in the interior as opposed to the fingered crack or fold in 3(e), which spans a significant fraction of the specimen width. The crack or fold feature is barely open in places and loosely welded to the substrate below it. A subsequent sliding pass in the same direction causes these features to shear off as needle-shaped lamellae.

Plane-strain finite element analysis (FEA) was performed using ABAQUS explicit dynamics [14] to explain fold formation. Slip line fields $[15,16]$ and one-dimensional approximations [17] are inadequate for complex plastic flows. Molecular dynamics, while useful for nanoscale tribological studies [18,19], is computationally infeasible at the mesoscale.
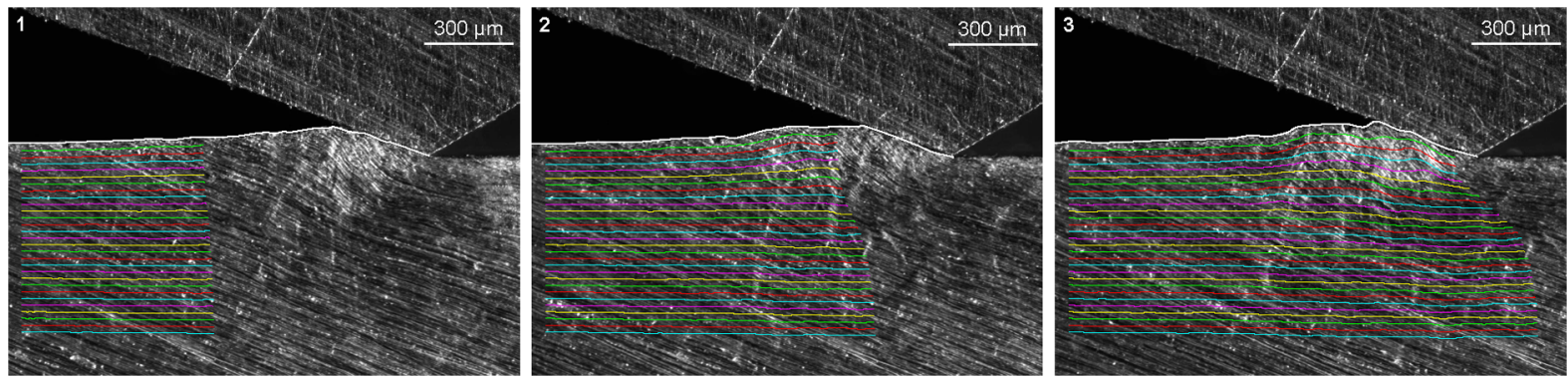

FIG. 2 (color online). Laminar flow, with a triangular prow ahead of the interface for $\alpha=-70^{\circ}$. Minor bumps produced ahead of the wedge on the surface are not amplified and do not interact to generate mesoscale folds. Frames 1-3 were selected from a highspeed image sequence and cover $1.0 \mathrm{~s}$ of real time. 


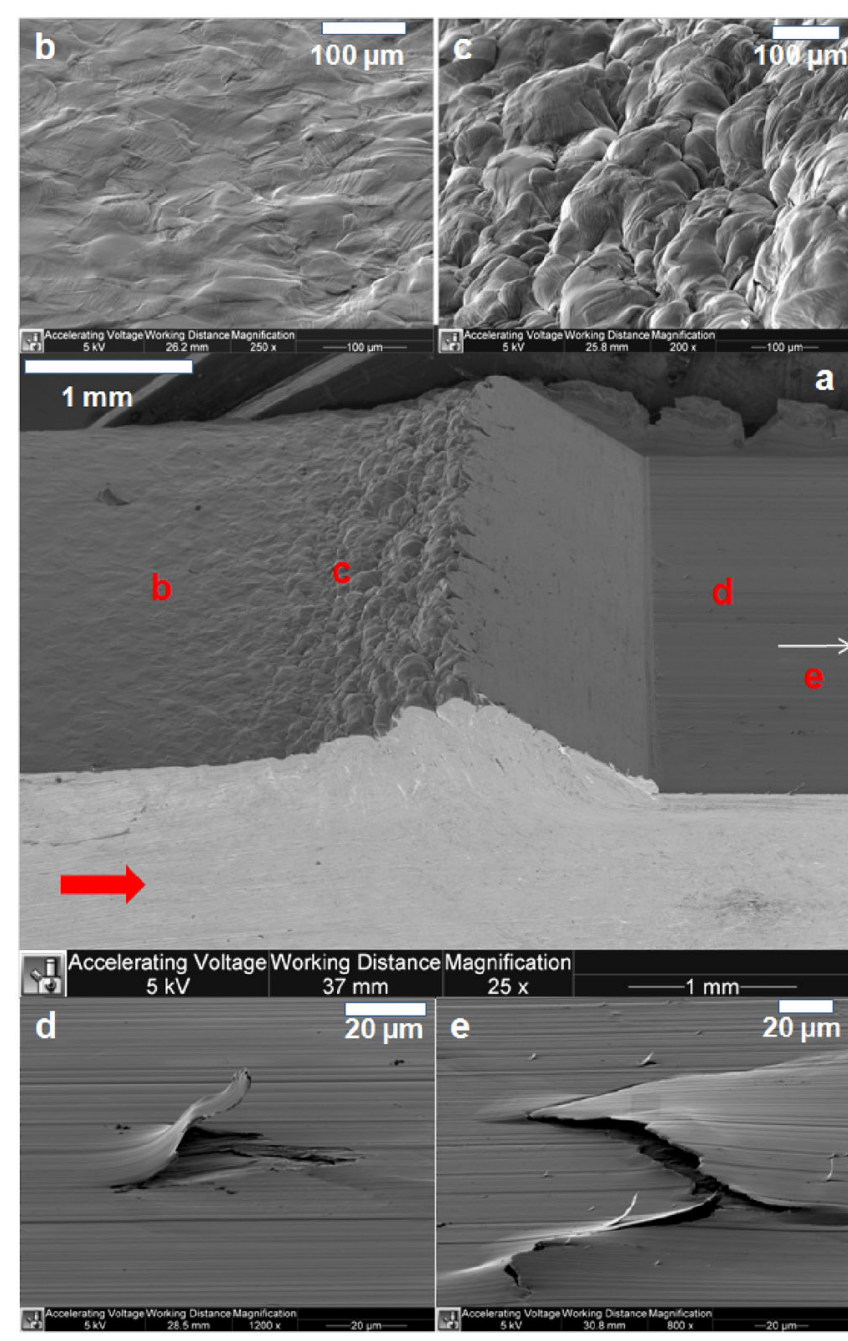

FIG. 3 (color online). Scanning electron micrographs of the sliding interface and vicinity at $\alpha=-60^{\circ}$. (a) Low magnification overview. High magnification subfigures (b)-(e) show surface features, and the red letters b-e within subfigure (a) indicate their approximate locations. Slip bands are clearly visible in the bumps in (b) and (c), and there is a clear growth in the bumpiness going from location $b$ to $c$, closer to the wedge face. (d) shows an isolated tear, and (e) shows an open crack or fold feature in the wake of the wedge. The red arrow indicates the workpiece sliding direction. Location e lies outside subfigure (a), as indicated.

Simulations with a homogeneous specimen produce laminar flow with $\alpha=-60^{\circ}$. While simulation with a sinusoidally perturbed workpiece surface of amplitude $\approx 0.05-0.1 h_{0}$ generated folds, experiments show that fold formation persists even when highly polished work surfaces with roughness amplitudes as small as $0.0002 h_{0}$ are used. Since polycrystalline $\mathrm{Cu}$ was used in experiments, heterogeneity due to grains with different mechanical properties was considered as an alternative cause. This hypothesis is supported by studies showing strong orientation dependence of flow stress and elastic properties $[20,21]$ in $\mathrm{Cu}$ single crystals. To this end, the FEA mesh

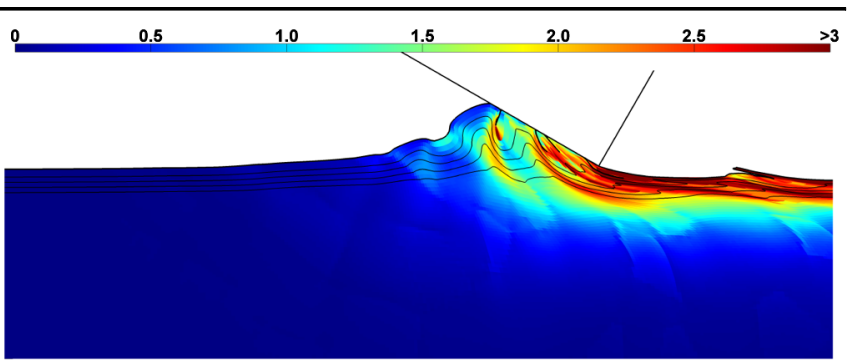

(a)
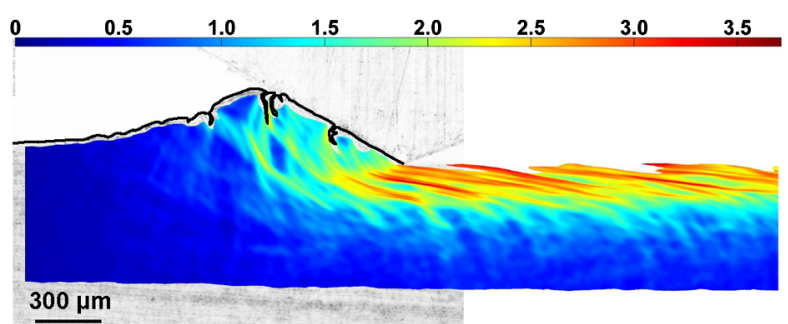

(b)

FIG. 4 (color online). Plastic strain in the sliding region for $\alpha=-60^{\circ}$. (a) FEA simulation showing a plastic instabilitydriven bump, surface fold, and crack development. (b) Measured plastic strain at $t=9.0 \mathrm{~s}$, superimposed on a background raw image taken at the same instant. The inclined, fingerlike protrusions are high-strain regions generated after folds pass the wedge tip.

of the workpiece was subdivided into convex cells using Voronoi subdivision. Two "phases," corresponding to elastically identical but plastically softer and harder $\mathrm{Cu}$, were assigned to these cells.

The FEA plastic strain field in Fig. 4(a) shows that this relatively simple model reproduces the bumps, folds, and shallow cracks observed in experiments. The mechanism of bump formation is as follows: the stress field ahead of the wedge face causes soft phases exposed on the surface to experience a necking-type plastic instability [22]. Note that this "necking" can occur even in the presence of lateral compressive or triaxial (nonhydrostatic) stresses [23,24]. This is confirmed by examining the von Mises stress $\sigma_{\mathrm{vm}}$ in the cell with softer material at the instant the deformation becomes unstable; one finds $\sigma_{\mathrm{vm}} \approx \sigma_{\text {instability,soft }} \ll$ $\sigma_{\text {instability,hard}}$. Also, $d \sigma_{\mathrm{vm}} / d \epsilon_{\mathrm{vm}} \approx \sigma_{\mathrm{vm}}$ under these conditions [23]. The softer cell experiences pinching and stretching in orthogonal directions due to neighboring hard cells during this process.

While constrained by harder neighboring regions, the softer phase is free to flow upward at the surface and produce initial bump(s). Bumps then come in contact with each other or the wedge face to produce a fold. Compressive stress promotes interaction between bumps by reducing the spacing between them as they travel toward the wedge face. The simulation shows that, for a "simple" fold, the fold length is roughly equal to the peak-to-peak bump amplitude; however, this length is larger for multiple interacting bumps. Bump and fold 
formation closely mirrors the experimental results in Fig. 1. Figure 4(b) shows measured plastic strain. The inclined, high-strain features in the wake of the wedge in Fig. 4(b) are adjacent to the crack or fold features mentioned previously and extend further into the depth. The crack or fold feature is also visible in the wake of the wedge in FEA in Fig. 4(a).

For a surface with initial sinusoidal roughness, the roughness and heterogeneity interact to produce longer folds and more "complex" folds in simulations. Significant folding does not occur when $\alpha=-70^{\circ}$ under otherwise identical conditions to $\alpha=-60^{\circ}$ simulations. This is on account of the lower strength of the compressive stress field ahead of the wedge when $\alpha=-70^{\circ}$. Note that rotational plasticity mechanisms that promote formation of micron-scale dislocation structures near hard particle interfaces [25] cannot explain mesoscale folding.

Plastic folds are quite different from the sulci generated in elastic membranes [26] and soft materials [27]. There, fold formation is buckling-driven and scale-free [27], while plastic fold formation critically depends on a scale parameter $\eta$, the ratio of the average grain size to a characteristic physical dimension (e.g., the prow height). There is a range of $\eta$ outside of which folding does not occur. Simulations show that fold amplitudes reduce for smaller $\eta, \approx 0.3$. Fold length is intimately connected to grain size and of the same order, even allowing for interactions with neighbors or preexisting roughness. Larger $\eta$ indicate greater mean interbump spacing and fewer opportunities for neighboring bumps to stay in the prow to form folds. However, a single bump may still interact with the wedge face to produce a fold. Experiments show that, in addition to $\alpha$ and $\eta$, adequate material ductility is necessary for folding [28]. Folding, however, occurs regardless of lubrication. A comprehensive experimental study of grain size effects is planned for the future.

Folding has implications for areas as diverse as surface generation, wear, and geology. Various processes based on repeated sliding and abrasion have been suggested as means of generating ultrafine grained and graded microstructures [4,5]. Our work shows that, without careful selection of tool incidence angle, surfaces produced using such methods will be damaged. Even the minor bumps observed at $\alpha=-70^{\circ}$ are undesirable from this perspective. Further, the occurrence of folding on surfaces with preexisting roughness fundamentally limits the quality of surfaces created by sliding.

Since the sliding wedge is a model asperity [16], our work suggests a new mechanism for crack formation in delamination wear, which also involves acute angle cracks. A single sliding pass of a suitably inclined asperity might produce a crack via fold formation instead of crack nucleation via accumulation of plastic strains over many cycles $[12,29]$. Heterogeneity- or roughness-driven folding might also contribute to ripples seen on the back surfaces of chips created in machining and fold-type features in ductile rocks [30].

Lastly, the observation of complex folds and vortexlike structures poses an interesting question related to turbulence. In fluids, the onset of turbulence involves vortices producing daughter vortices of smaller length scales as the Reynolds number increases and inertial effects begin to dominate viscous ones [7]. The highly dissipative nature of metal plasticity and greater resistance to deformation likely prevents incipient vortex structures from going on to this stage in copper, confining it to the very early stages of the onset of turbulence.

NSF Grants CMMI No. 1130525 and No. 1100712, U.S. Army Research Office Grant No. W911NF-12-10012, and General Motors are acknowledged for support. N.S. and Y. G. contributed equally to this work.

*nsundara@purdue.edu

†guo24@purdue.edu

[1] E. M. Kopalinsky and P. L. B. Oxley, Wear 190, 145 (1995).

[2] J. S. Courtney-Pratt and E. Eisner, Proc. R. Soc. A 238, 529 (1957).

[3] N. N. Zorev, Metal Cutting Mechanics (Pergamon, Oxford, UK, 1966).

[4] D. A. Hughes and N. Hansen, Phys. Rev. Lett. 87, 135503 (2001).

[5] T. H. Fang, W.L. Li, N. R. Tao, and K. Lu, Science 331, 1587 (2011).

[6] J. H. Dautzenberg and J. H. Zaat, Wear 23, 9 (1973).

[7] P. A. Davidson, Turbulence: An Introduction for Scientists and Engineers (Cambridge University Press, Cambridge, UK, 2004).

[8] D. A. Rigney and S. Karthikeyan, Tribol. Lett. 39, 3 (2010).

[9] See Supplemental Material at http://link.aps.org/ supplemental/10.1103/PhysRevLett.109.106001 for methods.

[10] R. J. Adrian, Annu. Rev. Fluid Mech. 23, 261 (1991).

[11] T. G. Murthy, C. Huang, and S. Chandrasekar, J. Phys. D 41, 074026 (2008).

[12] K. L. Johnson, Wear 190, 162 (1995).

[13] A. J. Black, E. M. Kopalinsky, and P. L. B. Oxley, J. Phys. D 26, 1892 (1993).

[14] ABAQUS Analysis User Manual, Dassault Systemes, Providence, RI, 2011.

[15] N. Bay, T. Wanheim, and A. S. Petersen, Wear 34, 77 (1975).

[16] J. M. Challen and P. L. B. Oxley, Wear 53, 229 (1979).

[17] S. Karthikeyan, H. J. Kim, and D. A. Rigney, Phys. Rev. Lett. 95, 106001 (2005).

[18] D. A. Rigney, X. Y. Fu, J. E. Hammerberg, B. L. Holian, and M.L. Falk, Scr. Mater. 49, 977 (2003).

[19] Y. Mo, K. T. Turner, and I. Szlufarska, Nature (London) 457, 1116 (2009).

[20] Y. Kawasaki and T. Takeuchi, Scr. Metall. 14, 183 (1980). 
[21] S. N. Dub, Y. Y. Lim, and M. M. Chaudhri, J. Appl. Phys. 107, 043510 (2010).

[22] W. A. Backofen, Deformation Processing (AddisonWesley, Reading, MA, 1972).

[23] H.L. D. Pugh and D. Green, Proceedings - Institution of Mechanical Engineers 179, 415 (1964).

[24] J. Chakrabarty, in Proceedings of the 13th International MTDR Conference, Birmingham, UK, 1972 (Macmillan, London, 1972), p. 565.
[25] L. M. Brown, Phil. Trans. R. Soc. A 355, 1979 (1997).

[26] L. Pocivavsek, R. Dellsy, A. Kern, S. Johnson, B. Lin, K. Y. C. Lee, and E. Cerda, Science 320, 912 (2008).

[27] E. Hohlfeld and L. Mahadevan, Phys. Rev. Lett. 106, 105702 (2011).

[28] Folding and vortex development are also observed in $\mathrm{Pb}$ and partially hardened $\mathrm{Cu}$.

[29] N. P. Suh, Wear 44, 1 (1977).

[30] P. J. Hudleston, Tectonophysics 16, 1 (1973). 